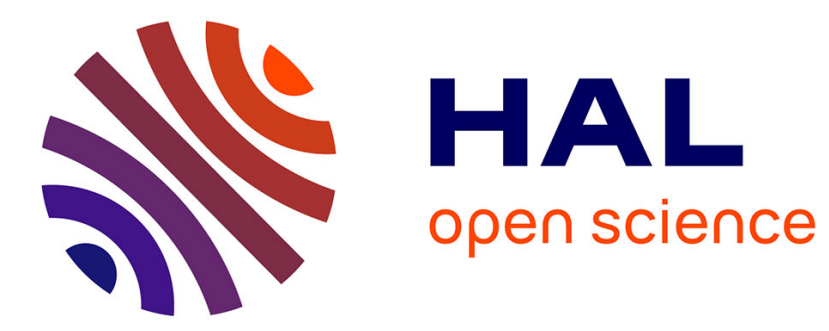

\title{
LA CODIFICATION OU LA DEMOCRATISATION DU DROIT
}

Sophie Lamouroux

\section{To cite this version:}

Sophie Lamouroux. LA CODIFICATION OU LA DEMOCRATISATION DU DROIT. Revue française de droit constitutionnel, 2001, 48, pp.801-824. hal-01236537

\section{HAL Id: hal-01236537 \\ https://hal-amu.archives-ouvertes.fr/hal-01236537}

Submitted on 1 Dec 2015

HAL is a multi-disciplinary open access archive for the deposit and dissemination of scientific research documents, whether they are published or not. The documents may come from teaching and research institutions in France or abroad, or from public or private research centers.
L'archive ouverte pluridisciplinaire HAL, est destinée au dépôt et à la diffusion de documents scientifiques de niveau recherche, publiés ou non, émanant des établissements d'enseignement et de recherche français ou étrangers, des laboratoires publics ou privés.

$$
\text { Copyright }
$$




\section{LA CODIFICATION OU LA DEMOCRATISATION DU DROIT}

Sophie Lamouroux

\section{INTRODUCTION}

De nos jours le stock législatif est estimé à près de 8000 textes dont le volume ne cesse d'augmenter, auquel il faut joindre les quelques 90000 textes réglementaires en vigueur ${ }^{1}$. Cette inflation législative répond aux exigences d'une société moderne, de plus en plus technique et complexe, dans laquelle chaque situation nouvelle appelle une règle de droit adaptée traduisant ainsi la conception actuelle du rôle de l'Etat.

A cette prolifération s'ajoute une «transformation qualitative $d u$ contenu de la norme $»^{2}$. Au mépris du principe de séparation des domaines législatif et réglementaire, le législateur précise minutieusement les conditions d'exécution des lois en multipliant les critères, paramètres ou éléments de la procédure afin d'éviter toute interprétation différente de la part de l'administration ou du juge car un «dispositif détaillé devient ainsi un moyen pour garantir une mise en auvre correcte, c'est-à-dire conforme à l'intention de ceux qui l'[1a loi] ont rédigée $»^{3}$. En outre l'examen parlementaire traduit parfois l'influence de revendications catégorielles ôtant à la loi son caractère général. La loi, ainsi fragilisée, se dévalorise et devient rapidement opaque et obsolète, ce qui invite le législateur à intervenir à nouveau et relance le mécanisme d'inflation.

Dès lors, l'écart grandissant entre d'une part, un droit prolifique, changeant et dont les conditions d'élaboration se détériorent et d'autre part, un citoyen censé, selon l'adage, ne rien ignorer des règles qu'il lui devient peu à peu impossible de connaître, est source d'inégalités. En 1991, le Conseil d'Etat dénonçait ce risque: "Si l'on n'y prend pas garde, il y aura demain deux catégories de citoyens : ceux qui auront les moyens de s'offrir les services des experts pour détourner ces subtilités à leur profit, et les autres, éternels égarés du labyrinthe juridique, laissés-pour-compte de l'Etat de droit $»^{4}$.

\section{A. - Les objectifs assignés à la codification}

Dans cet indéchiffrable contexte, la codification semble être une des solutions possible afin de rendre à la norme plus de clarté et de compréhension garantissant alors son unicité et sa cohérence et partant, un plein accès du droit pour tous.

\footnotetext{
${ }^{1}$ Cité in rapport de M. Alain Vidalies, au nom de la Commission des lois de l'Assemblée nationale, session ordinaire 1999-2000, Doc. A.N., n 1917, p. 5.

2 ALBERTINI (P.), « La codification et le Parlement », A.J.D.A., 1997, p. 662.

${ }^{3}$ Ibid.

${ }^{4}$ Conseil d'Etat, Rapport public 1991, Paris, La Documentation française, Etudes et Documents, n 43, $1992, \mathrm{p}$. 21.
} 
Elle a pour objectif principal d'effectuer une mise en ordre du droit ${ }^{5}$. Ce travail de recensement systématique des textes en vigueur dans une matière donnée est difficile. En effet il faut identifier parmi la succession de textes ceux qui ont été abrogés et ceux qui s'appliquent. De cette rationalisation du droit découlent plusieurs conséquences : la clarté, la cohérence et la simplification. Le droit devient clair parce qu'à la place d'un droit éparpillé, on trouve un droit rassemblé matériellement dans un même texte ou plusieurs mais toujours selon un regroupement logique et systématique. La cohérence se manifeste ensuite par l'unité de style et de pensée dans la mesure où le code est organisé selon un plan logique et ordonnancé. Enfin simplifier le droit signifie rendre sa connaissance plus aisée en en supprimant les contradictions, les expressions désuètes, les répétitions, en le purgeant de ses éléments d'opacité car, "plus un droit devient complexe et abondant, plus il devient inintelligible, secret et donc arbitraire et injuste, plus il devient un obscur message codé, et c'est par un code qu'il est le mieux décodé » ${ }^{6}$.

Par suite, l'ordonnancement du droit poursuit un but majeur, celui de le rendre plus largement accessible. Si les textes concernant un domaine donné sont clairs, simples, ordonnés et répertoriés dans un ensemble cohérent, alors la formule selon laquelle « Nul n'est censé ignoré la loi » recouvre un sens. Il en résulte ainsi un sentiment de sécurité car on a l'assurance d'avoir l'ensemble des textes en vigueur sur le sujet. Mais il ne faudrait pas se méprendre, la codification est un des moyens parmi d'autres pour atteindre cette compréhension de la norme qui constitue un des éléments de l'Etat de droit et non un remède miracle $^{7}$. Elle favorise seulement l'accès au droit et son application, en contribuant à un usage facilité de la norme.

Toutes les formes de codification à travers 1 'histoire ${ }^{8}$ ont pour justification commune la «volonté d'avoir un droit rationnel et accessible » ${ }^{9}$ dont le résultat aboutit à un droit codifié. En revanche une distinction peut être faite en ce qui concerne l'objet de la codification et qui oppose deux catégories : la codification qui réforme et celle qui reforme ${ }^{10}$.

La première est une codification-modification ${ }^{11}$ où le codificateur élabore un droit le plus accompli possible. Mais il ne s'agit pas d'un droit pleinement innovateur aboutissant à

\footnotetext{
${ }^{5}$ BRAIBANT (G.), «La problématique de la codification », R.F.A.P., 1997, n 82, pp. 167 et ss. ; «Utilité et difficultés de la codification », Droits, 24-1996, pp. 64 et ss. ; «Codifier : Pourquoi ? Comment ?», R.F.A.P., janvier-mars $1995, n^{\circ} 73$, pp. 129 et ss.

${ }^{6}$ MALAURIE (P.), « Les enjeux de la codification », A.J.D.A., 1997, p. 644.

${ }^{7}$ En ce sens voir, ALBERTINI (P.), « La codification et le Parlement », A.J.D.A., 1997, p. 661.

${ }^{8}$ Pour une présentation historique des entreprises de codification en France et en Europe voir : GAUDEMET (J.), Les naissances du droit. Le temps, le pouvoir et la science au service du droit, Paris, Montchrestien, 2è éd., 1999, pp. 178 et ss.

${ }^{9}$ BRAIBANT, (G.), «Utilité et difficultés de la codification », précité, p. 62.

${ }^{10}$ L'expression est de Guy Braibant qui, selon la même idée distingue également les codifications raciniennes (le droit tel qu'il est) et cornéliennes (le droit tel qu'il devrait être) (ibid., p. 63). De la même manière Jeremy Bentham opposait The Law as it is et The Law as it ought to be ; en latin on parle de codification de lege lata et de lege ferenda.

${ }^{11}$ Expression citée in MALAURIE (P.), «Peut-on définir la codification? Eléments communs et éléments divers », R.F.A.P., 1997, $\mathrm{n}^{\circ}$ 82, pp. 179 et s. ; « Rapport de synthèse », in La codification, Actes du colloque
} 
une réforme globale du droit puisque « cette codification réformatrice est une combinaison de la reprise du droit existant et d'un ensemble de réformes, les deux étant complémentaires $»^{12}$. Elle réécrit en les modifiant, parfois profondément, ou en les complétant, les dispositions existantes en édictant des règles nouvelles. Cette codification ambitieuse est difficile à réaliser en ce sens qu'elle nécessite un accord - qu'il est peu aisé d'obtenir - entre les autorités compétentes, le Gouvernement et le Parlement.

La seconde est une codification non modificative appelée également «à droit constant ». Elle n'innove pas mais regroupe et ordonne les textes écrits existants c'est-à-dire en vigueur lors de son approbation et officiellement publiés par l'autorité publique. Elle ne se contente pas uniquement de compiler les textes mais elle les adapte en fonction d'un certain nombre de principes - sur lesquels nous reviendrons - sans modification de fond.

Inévitablement le principe de la codification a suscité des objections ${ }^{13}$. On lui reproche tout d'abord une perte d'histoire, laquelle permet d'expliquer et de comprendre le droit et son évolution. Afin de pallier l'absorption des lois anciennes par les codes, des codes de tables de concordance existent et ont pour effet de situer l'article en cause dans son contexte historique. On lui reproche ensuite une perte de poésie en raison de la suppression de textes originaux mais désuets «jamais appliqués ou devenus inapplicables » ${ }^{14}$. Mais c'est surtout la lisibilité du texte qui prime et la disparition de termes archaïques, quoique pittoresques, améliore cette compréhension. Enfin la codification figerait le droit. Cet argument, de loin le plus grave, est difficilement soutenable car il signifierait que les codificateurs auraient le sentiment de faire œuvre parfaite. Ce qui n'est pas le cas. Certes, la codification apporte une certaine stabilité mais elle ne gèle pas l'évolution du droit. Elle facilite au contraire la réforme en devenant son point de départ ${ }^{15}$. Si matériellement les textes dans un domaine particulier sont rassemblés et ordonnés, il devient plus facile alors d'entreprendre des modifications qui auront dès lors une cohérence juridique et technique.

\section{B. - Une préoccupation ancrée dans l'histoire}

La pratique de la codification dans l'histoire et dans divers pays a revêtu des visages différents, tenant compte des réalités attachées à chacune des cultures ${ }^{16}$. Mais la volonté de

organisé les 27 et 28 octobre 1995 par l'Ordre des avocats du barreau de Toulouse et l'Institut d'études judiciaires de la Faculté de droit de Toulouse, Paris, Dalloz, 1996, pp. 197-208.

${ }^{12}$ BRAIBANT, (G.), «Utilité et difficultés de la codification », précité, p. 63.

${ }^{13}$ GUY (S.), «Une utopie : la codification », R.F.D.C., 26-1996, pp. 273-310 ; « De la codification », L.P.A., 12 mars 1997, n 31, pp. 31-17.

${ }^{14}$ BRAIBANT, (G.), «Utilité et difficultés de la codification », précité, p. 66. Afin d'illustrer son propos, l'auteur fait référence à une loi du 28 août 1792 sur les «terres vaines et vagues de Bretagne » inappliquée et ignorée dont l'abrogation n'a soulevé aucune difficulté.

${ }^{15}$ BRAIBANT (G.), «Codifier : Pourquoi ? Comment? », précité, p. 131.

16 Pour une présentation des codifications les plus symptomatiques entreprises à travers l'histoire voir: GAUDEMET (J.), « La codification justinienne », R.F.A.P., avril-juin 1997, $\mathrm{n}^{\circ}$ 82, pp. 233-238; MOUSSA (L. 
collecter et d'améliorer le droit existant dans un esprit de système a toujours inspiré les projets de codification. Hammourabi, Justinien, l'Eglise ou Napoléon ont tous agi dans ce sens.

En France ${ }^{17}$, la codification a emprunté plusieurs voies. Tour à tour codification à droit constant et codification modificative ont marqué le cheminement d'un processus qui depuis 1989 semble stabilisé.

La nécessité d'une connaissance méthodique des textes en vigueur est apparue au XVIe siècle ${ }^{18}$, sous Henri III. Il fut décidé, à la suite des Etats généraux de Blois en 15761577 , d'établir un regroupement complet des édits et des ordonnances dans un code unique afin de mettre de l'ordre dans un droit devenu confus. Cette compilation fut confiée à un conseiller d'Etat, Barnabé Brisson mais c'est sous le nom de Code Henri III que ce premier effort de codification est connu. Plus tard Colbert avec ses grandes ordonnances, qui ne sont pas formellement dénommées codes, aura une ambition identique : celle de rassembler dans un même ensemble des textes relatifs à la procédure civile, pénale, aux eaux et forêts, à la marine et au commerce. A la différence du Code Henri III, ces ordonnances ont constitué des textes novateurs modifiant le droit existant. C'est la même volonté qui a guidé les hommes de la Révolution, du Consulat et de l'Empire pour l'élaboration du Code civil, du Code pénal, du Code de procédure pénale, du Code de commerce et du Code d'instruction criminelle. La modernité attachée à cette adaptation du droit existant donnera un retentissement majeur à l'unification du droit entreprise par Napoléon à travers l'Europe.

La codification va ensuite connaître près d'un siècle et demi de mise en sommeil ponctué par quelques tentatives éparpillées en dehors de toute entreprise d'ensemble. Le Code rural, le Code du travail, le Code de la sécurité routière ou encore le Code de la famille et quelques codes particuliers dont le Code du travail maritime ou le Code disciplinaire et pénal de la marine marchande verront le jour au cours de cette période mais il s'agit plus de la compilation de textes existants que d'un véritable effort de codification. Au contraire on assiste à une décodification notamment du Code de commerce qui avait 650 articles sous l'Empire et qui ne contient plus aujourd'hui que 150 articles dans la mesure où la plupart les grandes lois du droit commercial ont été faites hors du code.

Ce n'est qu'après la Seconde guerre mondiale que le mouvement de codification repart de façon systématique et rationnelle. Le décret $n^{\circ} 48-800$ du 10 mai 1948 a institué une commission supérieure chargée d'étudier la codification et la simplification des textes législatifs et réglementaires. Menée par un inspecteur des finances, Gabriel Ardant proche collaborateur de Pierre Mendès-France, et émanant d'un organe administratif (le comité

F.), «La codification du droit musulman », R.F.A.P., avril-juin 1997, n 82, pp. 249-255; TOXE (P.), « La codification en droit canonique $»$, R.F.A.P., avril-juin 1997, $\mathrm{n}^{\circ}$ 82, pp. 239-247.

${ }^{17}$ Pour une présentation historique de la codification en France voir : SUEL (M.), Essai sur la codification à droit constant. Précédents - Débuts - Réalisation, Paris, Journal officiel de la République française, 2è éd., 1995, $296 \mathrm{p}$.

${ }^{18}$ La connaissance méthodique du droit devient à cette époque de plus en plus pressante car depuis l'ordonnance de Montils-lès-Tours en 1453 mettant par écrit les coutumes, et le règne de Charles VII (1422-1461) au cours duquel abondent les ordonnances royales, il existe davantage de textes en vigueur, dans différents domaines. Dès lors devant leur multiplication en nombre et en importance, il apparaît nécessaire de les connaître, de savoir s'ils sont appliqués ou non, en vigueur ou abrogés. 
central pour le coût et le rendement des services publics), cette démarche est avant tout une réforme administrative plus qu'une mise en ordre du droit. Cette codification ne naît pas dans le monde des juristes mais dans celui de ceux qui ont des liens avec l'économie et qui se préoccupent de l'organisation pratique du travail au sein des administrations et des entreprises ${ }^{19}$. Au désir d'obtenir des règles claires et complètes s'ajoute la volonté d'efficacité dans la recherche des meilleurs résultats et du moindre coût. Entre 1948 et 1988, une quarantaine de codes sera adoptée dans différents domaines (économique, social, administratif).

Cependant cette codification trouve ses limites dans la méthode utilisée qui favorise un sentiment d'incertitude autour de la norme applicable et, dès lors, devient source de contentieux et de précarité pour l'usager. Elle est en effet qualifiée d'administrative dans la mesure où il s'agit d'une codification sanctionnée par décret parfois ratifié ultérieurement par le Parlement: «ayant une nature réglementaire, les articles nouvellement codifiés ne pouvaient en conséquence abroger les dispositions législatives initiales. Lorsqu'ils étaient modifiés ultérieurement par le législateur, qui leur reconnaissait ainsi implicitement valeur législative, subsistaient alors à la fois la disposition législative initiale et la disposition codifiée et modifiée $»^{20}$. Ainsi le juge confronté à un doute quant à la norme applicable, a dû faire prévaloir la rédaction initiale ${ }^{21}$. Pour remédier à cette situation inconfortable, le législateur est intervenu pour valider d'abord plusieurs codes adoptés par voie réglementaire ${ }^{22}$ puis ponctuellement à partir des années $70^{23}$.

\section{C. - Les principes établis depuis 1989}

1989 marque la date de ce qu'on a appelé « la relance de la codification », à l'initiative du Premier ministre, Michel Rocard secondé par son conseiller le Professeur Guy Carcassonne et par Guy Braibant. La codification s'insère dans un vaste mouvement de réforme de l'Etat ${ }^{24}$ visant à rendre ce dernier plus simple dans ses textes et procédures, plus proche des citoyens et des entreprises et plus efficace ${ }^{25}$. Le décret de base de 1948 est modifié

\footnotetext{
${ }^{19}$ GROSSHENS (J.-C.), « De la codification par décret des lois et des règlements », D., 1958, Chr. XXIII, p. 157.

${ }^{20}$ Voir rapport de M. Alain Vidalies, au nom de la Commission des lois de l'Assemblée nationale, précité, p. 7.

${ }^{21}$ Voir C.E., 13 septembre 1995, Ministre du budget c/ Mme Anna Simha, Lebon, req. 126830 ; A.J.D.A., 1996, p. 65, note V. Haïm ; voir également les arrêts cités in Rapport de M. Patrice Gélard, au nom de la Commission des lois du Sénat, session ordinaire 1999-2000, Doc. S., nº 4, p. 7 : C.Cass., 23 janvier 1989 et C.E., 22 mai 1989.

${ }^{22}$ La loi du 3 juin 1958 valida quinze codes.

${ }^{23}$ Le Code de l'urbanisme est validé en 1976, le Code de la sécurité sociale en 1987 et celui de la voirie routière en 1989. Certains codes tels que le Code du service national, de la nationalité ou de la justice militaire n'ont jamais été validés.

${ }^{24}$ SILICANI (J.-L.), « La codification : instrument de réforme de l'Etat en France », A.J.D.A., 1997, pp. 640641.

${ }^{25}$ Voir circulaire du 26 juillet 1995 du Premier Ministre relative à la préparation et à la mise en œuvre de la réforme de l'Etat et des services publics, J.O.R.F., 28 juillet 1995, p. 11217.
} 
par celui du 12 septembre $1989^{26}$ qui renforce la Commission de codification ${ }^{27}$. Désormais, le Premier ministre la préside et appuie de son autorité morale l'effort de codification alors qu'auparavant le président était un ministre ou un secrétaire d'Etat rattaché aux réformes administratives ou à la fonction publique qui n'avait aucun poids à l'égard des grands ministères. Le vice-président de la Commission, qui assure la présidence de fait est un président de section au Conseil d'Etat, en activité ou honoraire (actuellement Guy Braibant); elle est en outre composée de membres de la Cour de cassation, du Conseil d'Etat, de la Cour des comptes et de six directeurs d'administration centrale ${ }^{28}$ auxquels se joignent les directeurs des administrations centrales concernées par les projets de codification. Le Parlement est également associé puisque la Commission des lois de chaque assemblée nomme un représentant permanent au sein de la Commission. Elle dispose d'un véritable état major qui assiste la présidence et renforce l'institution : il est composé d'un secrétariat, d'un rapporteur général, de deux rapporteurs généraux adjoints et d'une quinzaine de rapporteurs particuliers attachés à la rédaction des codes. Pour accomplir sereinement sa tâche, la Commission, qui siège à Matignon, bénéficie de moyens financiers et de crédits.

Afin de mener à bien le processus de codification, la Commission a dégagé deux grands principes sur lesquels repose sa méthode de travail : la codification doit être effectuée à droit constant et doit faire l'objet, pour les parties législatives des codes, d'une validation législative.

Codifier à droit constant signifie codifier sans modifier, sans réformer ${ }^{29}$. Si avant 1989 cela se justifiait parce que les codes prenaient la forme de décrets, depuis, cette obligation découle de la ratification parlementaire. Les dispositions codifiées sont celles qui sont en vigueur au moment du dépôt du projet de loi devant le Parlement, en sont donc exclues les sources contractuelles, jurisprudentielles et coutumières. La codification à droit constant ayant pour objet d'appréhender seulement les textes édictés par les autorités nationales, ceux émanant d'une autorité non nationale en sont exclus. En effet, si le Parlement n'a pas le pouvoir d'abroger une disposition, celle-ci ne saurait figurer dans la partie législative du code. Il en va de même pour le Gouvernement et la partie réglementaire. Aucune norme

\footnotetext{
${ }^{26}$ Décret $\mathrm{n}^{\circ}$ 89-647 du 12 septembre 1989 relatif à la composition et au fonctionnement de la Commission supérieure de codification, J.O.R.F., 13 septembre 1989, p. 11560.

${ }^{27}$ BRAIBANT (G.), «La commission supérieure de codification», in La codification, Actes du colloque organisé les 27 et 28 octobre 1995 par l'Ordre des avocats du barreau de Toulouse et l'Institut d'études judiciaires de la Faculté de droit Toulouse, Paris, Dalloz, 1996, pp. 97-105 ; ROBINEAU (Y.), «Les structures françaises : la Commission supérieure de codification », R.F.A.P., avril-juin 1997, pp. 263-268.

${ }^{28}$ Le directeur des affaires civiles et du sceau, le directeur des affaires criminelles et des grâces, le directeur général de l'administration et de la fonction publique, le directeur au secrétariat général du gouvernement, le directeur des Journaux officiels et le directeur aux affaires politiques, administratives et financières au secrétariat d'Etat à l'outre-mer.

${ }^{29}$ BRAIBANT (G.), « Codifier : Pourquoi ? Comment ? », précité, pp. 127-141 ; DUFOUR (O.), BONNET (E.), «Codifier pour mieux réformer. Un entretien avec Guy Braibant, vice-président de la Commission supérieure de codification », L.P.A., 21 novembre 1997, $\mathrm{n}^{\circ}$ 140, pp. 5-11.
} 
internationale, et au premier chef européenne, ne peut apparaître dans un code ${ }^{30}$. Cependant, le premier rapport de la Commission précisait que figureraient en annexe les dispositions communautaires se rapportant à la matière codifiée. En outre, il ne s'agit pas d'une simple compilation mais d'une codification réfléchie et intelligente. En effet le principe du droit constant autorise la Commission à une mise à jour du langage en éliminant les expressions vieillies ou par trop pittoresques qui rendent le texte incompréhensible. Mais il s'agit surtout d'adapter des textes parfois anciens aux droit et principes constitutionnels actuels dans le respect de la hiérarchie des normes. Cela permet à la Commission de procéder à des reclassements de dispositions codifiées dans la partie législative ou réglementaire ${ }^{31}$.

La validation législative est un principe issu des errements de la codification jusqu'en 1988. Le Parlement est ainsi directement associé à l'œuvre de codification et les dispositions intégrées au code, ayant force de loi, abrogent les lois antérieures. C'est un véritable progrès qui donne aux codes une pleine cohérence. Toutefois, si juridiquement ce principe est pleinement justifié, il n'en comporte pas moins des inconvénients. La validation législative n'a pas de signification politique et il ne s'agit pas de remettre en cause le contenu des codes mais de les améliorer sur un plan technique. Or les parlementaires ont du mal à se limiter à ce seul rôle. La validation présente ensuite l'inconvénient de scinder les codes en deux parties (législative et réglementaire) et donc d'opérer selon deux procédures distinctes en différant l'adoption de la partie réglementaire des codes. Enfin, et surtout, l'intervention du Parlement est dépendante de l'ordre du jour des assemblées et donc du Gouvernement.

C'est précisément ce dernier constat qui va pousser le Gouvernement, en 1999, à utiliser la voie des ordonnances de l'article 38 de la Constitution afin d'accélérer un processus bloqué et de surmonter l'engorgement du Parlement. La décision du Conseil constitutionnel consécutive à l'examen de la loi d'habilitation, est l'expression de la consécration de la codification. Plus encore, avec cette décision, le principe d'accessibilité et d'intelligibilité de la loi devient un objectif de valeur constitutionnelle et la codification, un des moyens de réaliser. Ainsi ce nouvel épisode d'une part enracine le processus de codification ainsi que les principes et méthodes de travail le concernant dans le droit constitutionnel français et, d'autre part, participe à la consolidation de l'Etat de droit.

\footnotetext{
${ }^{30}$ Les limites de cette orientation ont été soulignées à de nombreuses reprises, voir notamment : GUY (S.), «Une utopie : la codification », précité, pp. 273-310 ; «De la codification », précité, pp. 31-17; ROBINEAU (Y.), «A propos des limites d'une codification à droit constant », A.J.D.A., 1997, p. 655.

${ }^{31}$ Pour des précisions concernant les modalités de déclassement effectué par la Commission voir : Commission supérieure de codification, Neuvième rapport annuel, Paris, Les éditions du Journal officiel, 1998, pp. 44-45.
} 


\section{I. - LES ORDONNANCES DE L'ARTICLE 38 : UNE SOLUTION AU BLOCAGE DU PROCESSUS DE CODIFICATION}

Entre 1989 et 1996 le Parlement a adopté plusieurs codes selon la procédure et la méthode de travail introduites dès 1989 : le Code de la propriété intellectuelle, le Code de la consommation, les livres Ier, II, VI et VIII du Code rural, les livres Ier, II et III du Code des juridictions financières et le Code des collectivités territoriales. Au total ont été regroupées dans ces codes près de sept cents lois représentant un total de 12 à 15000 articles. En outre, la circulaire du 30 mai 1996 relative à la codification des textes législatifs et réglementaires ${ }^{32}$ constatant la prolifération et l'enchevêtrement des textes de notre ordre juridique, avait pleinement reconnu la place de la codification à droit constant dans le combat mené contre l'inflation législative. Plus précisément, elle avait pour objet d'une part de consacrer le principe de la codification à droit constant et d'autre part de préciser les modalités et les méthodes de programmation, de préparation et de rédaction des codes ainsi que les règles de fonctionnement de la Commission supérieure de codification. En outre, était annexé à cette circulaire le programme de codification adopté par la Commission supérieure de codification pour la période 1996-2000 prévoyant l'élaboration de 22 nouveaux codes et la refonte de 18 codes existants.

Malgré les incontestables progrès accomplis en matière de codification et la volonté du Gouvernement de la maintenir au rang de ses préoccupations essentielles, il faut malheureusement constater que l'élaboration des codes dans le contexte défini est dans l'impasse. Depuis la publication du Code général des collectivités territoriales en février 1996 et l'adoption définitive par le Parlement du livre VI du Code rural, aucun code n'a été adopté par le Parlement alors que selon le neuvième rapport de la Commission supérieure de codification $^{33}$, sept projets de code et deux livres du Code rural sont achevés à cette date et attendent d'être examinés soit par le Conseil d'Etat ${ }^{34}$, soit par le Parlement ${ }^{35}$.

Le blocage est essentiellement dû à l'encombrement de l'ordre du jour législatif avec pour conséquence une accumulation de codes prêts mais non adoptés. Les raisons de cette situation sont multiples. Tout d'abord, certains projets de loi de codification ont fait l'objet de critiques quant à la forme devant l'Assemblée nationale comme le Code de l'éducation et celui de l'environnement ou ont été tout simplement rejetés pour des raisons de fond comme le projet de Code de commerce. Ensuite, la codification s'effectuant à droit constant, sa signification politique est limitée. Le rapport de la Commission des lois de l'Assemblée

\footnotetext{
${ }^{32}$ Circulaire du 30 mai 1996 relative à la codification des textes législatifs et réglementaires, J.O.R.F., 5 juin 1996, p. 8263 ; J.C.P., 1996, G, 3953, note G. Drago, «Lois : de la codification à l'évaluation. Premiers commentaires de la circulaire du 30 mai 1996 et de la loi n 96-516 du 14 juin $1996 »$.

33 Commission supérieure de codification, Neuvième rapport annuel, Paris, Les éditions du Journal officiel, 1998, p. 7.

${ }^{34}$ Le Code de la santé publique et le Code de justice administrative.

${ }^{35}$ Le Code de l'éducation, le Code de l'environnement, le Code de la communication et du cinéma, le Code de commerce et le livre VII du Code rural.
} 
nationale consacré au projet de loi relatif aux droits des citoyens dans leurs relations avec les administrations présente un résumé significatif de la difficulté : «Il n'est guère attrayant, en effet, pour des élus d'examiner des projets complexes qui, parce qu'ils sont élaborés à droit constant, ne s'inscrivent pas dans le cadre d'un programme politique. Il s'agit même parfois pour le Parlement, compte tenu des changements de majorité, d'adopter, dans le cadre d'un code, des dispositions dont la modification est envisagée ${ }^{36}$. Dès lors, l'inscription à l'ordre du jour des parties législatives des codes n'est plus considérée, même s'il faut le regretter, comme une priorité par le Gouvernement, d'autant que le processus d'adoption est long, austère voire ingrat et constitue une surcharge de travail pour les services du Parlement.

Aussi le blocage de la phase parlementaire remet-il en cause le processus de codification et entraîne des conséquences dommageables constatées en ces termes par la Commission supérieure de codification dans son neuvième rapport :

«les projets de code une fois déposés sur le bureau d'une assemblée vieillissent rapidement $d u$ fait des réformes de fond envisagées entre temps (cas du code de la communication et du cinéma) ou du fait de l'intervention de lois nouvelles dans la matière considérée (cas du code de l'environnement) ce qui induit, soit le report de l'examen du projet, soit une mise à jour toujours délicate à opérer ;

le blocage d'un projet de code au stade parlementaire se répercute non seulement sur l'élaboration de la partie réglementaire dudit code mais encore sur l'élaboration des autres projets de code législatif avec lesquels il s'articule (code de commerce et code monétaire et financier) ;

l'ensemble de la chaîne participant à la confection de codes, des missions de codification jusqu'au Conseil d'Etat, se trouve affecté par le goulot d'étranglement que constitue l'absence de débouché parlementaire (cas du code rural)».

Projets obsolètes, répercussions sur la partie réglementaire ${ }^{37}$, arrêt du processus de codification, à cela il faut ajouter une démobilisation des intervenants, qu'il s'agisse de la Commission supérieure de codification, des services ministériels ou du Conseil d'Etat qui, pour sa part a renvoyé des projets de codes au Gouvernement sans les examiner pour protester contre une pratique en panne.

Le recours à la procédure de l'article 38 pour surmonter cette situation de blocage s'est ainsi peu à peu imposé. Lors des travaux préparatoires de la loi n 2000-231 du 12 avril 2000

\footnotetext{
${ }^{36}$ Rapport de Mme Claudine Ledoux au nom de la Commission des lois de l'Assemblée nationale, onzième législature, Doc. A.N., ${ }^{\circ} 1613$, pp. 16 et s.

${ }^{37}$ Il faut cependant ajouter qu'en dehors de tout blocage parlementaire, la parution de la partie réglementaire des codes intervient longtemps après la partie législative. Pour preuve, la partie Législative du Code des collectivités territoriales fut promulguée le 21 février 1996 alors que sa partie Réglementaire ne l'a été que le 7 avril 2000 (Décret $\mathrm{n}^{\circ}$ 2000-318 du 7 avril 2000 relatif à la partie réglementaire du Code général des collectivités territoriales, J.O.R.F., 9 avril 2000, p. 5469). Or la codification des actes réglementaires est aussi importante que celle du corpus législatif car les usagers doivent pouvoir disposer d'un texte d'ensemble afin que la compréhension du code soit pleine et entière.
} 
relative aux droits des citoyens dans leurs relations avec les administrations ${ }^{38}$, le Ministre de la fonction publique, de la réforme de l'Etat et de la décentralisation, s'agissant de l'examen de l'article $3^{39}$ du projet de loi, a fait part de l'intention du Gouvernement d'utiliser, pour l'adoption des codes actuellement en instance devant le Parlement ou dont l'examen par la Commission supérieure de codification est achevé, la procédure des ordonnances prévue par l'article 38 de la Constitution. Dans sa séance du 23 mars 1999, la Commission supérieure de codification s'est montrée favorable à cette solution pour débloquer le processus de codification.

En 1997, l'idée d'une validation législative par ordonnance avait déjà été envisagée par Jean-Ludovic Silicani, alors commissaire à la réforme de l'Etat. Ce dernier constatant l'encombrement de l'ordre du jour des assemblées préconisait cette solution : «L'une des pistes à envisager, après avoir été étudiée et présentée aux assemblées pour vérifier la pertinence de son principe et éventuellement de sa méthode, consisterait à prévoir chaque année qu'une loi habilite le gouvernement à codifier par ordonnance dans deux ou trois secteurs précis. Ces textes seraient ensuite validés par le Parlement ${ }^{40}$.

En conséquence, la loi du 16 décembre $1999^{41}$ habilite le Gouvernement à procéder par ordonnances, à l'adoption de la partie législative de neuf codes : le Code rural, le Code de l'éducation, le Code de la santé publique, le Code de commerce, le Code de l'environnement, le Code de la justice administrative, le Code de la route, le Code de l'action sociale et le Code monétaire et financier.

\footnotetext{
${ }^{38}$ Loi n ${ }^{\circ}$ 2000-231 du 12 avril 2000 relative aux droits des citoyens dans leurs relations avec les administrations, J.O.R.F., 13 avril 2000, p. 5646. Pour un commentaire de la loi, voir notamment : ARRIGHI DE CASANOVA (J.), «Une nouvelle étape de l'amélioration des relations entre l'administration et les citoyens : la loi "D.C.R.A." du 12 avril $2000 »$, R.F.D.A., 2000, pp. 724-736 ; BOULAY (J.), « La loi n ${ }^{\circ} 2000-321$ du 12 avril 2000 relative aux droits des citoyens dans leurs relations avec les administrations : une révolution en attente d'applications ? », Gazette de Palais, Dimanche 24 au Jeudi 28 décembre 2000, pp. 3-6 ; CHEVALLIER (J.), «La transformation de la relation administrative : mythe ou réalité ? (à propos de la loi n ${ }^{\circ} 2000-321$ du 12 avril 2000 relative aux droits des citoyens dans leurs relations avec les administrations) », D., 2000, n 38, Chr., pp. 575-584 ; DELAUNAY (B.), «La loi du 12 avril 2000 relative aux droits des citoyens dans leurs relations avec l'administration », R.D.P., 2000, pp. 1191-1238 ; FERRARI (P.), «Les droits des citoyens dans leurs relations avec les administrations. Commentaire général de la loi n 2000-321 du 12 avril $2000 »$, A.J.D.A., 2000, pp. 471485 ; PISSALOUX (J.-L.), «Relations des citoyens avec les administrations : le changement dans la continuité ( $1^{\text {ère }}$ partie) », L.P.A., 14 février 2001, $\mathrm{n}^{\circ} 32$, pp. 4-16; « Relations des citoyens avec les administrations : le changement dans la continuité (suite et fin)», L.P.A., 15 février 2001, n 33, pp. 5-11; PRETOT (X.), «L'amélioration des relations entre l'administration et ses usagers : la loi n ${ }^{\circ} 2000-321$ du 12 avril 2000, Bulletin fiscal Francis Lefebvre, 2000, ${ }^{\circ}$ 11, pp. 591-600; TREPPOZ (A.), « La loi du 12 avril 2000 sur les relations entre les citoyens et les administrations. Présentation générale », Dr. adm., Août-Septembre 2000, pp. 4-8.

${ }^{39}$ Cet article prévoyait dans sa rédaction initiale un programme d'adoption de codes avant la fin de la présente législature. Il était accompagné d'une annexe, établissant la liste des codes nouveaux et des codes à refondre. Le Sénat a supprimé cet article ainsi que l'annexe lors de l'examen en première lecture, le 10 mars 1999, au motif qu'un programme législatif de codification, sanctionné par une date butoir, constituait une injonction à légiférer, contraire au principe de la séparation des pouvoirs. En outre, il a considéré que le programme de codification était matériellement irréalisable, en raison de son ampleur et du délai imparti. Compte tenu des orientations prises par le Gouvernement, le rétablissement de l'article 3 dans sa rédaction initiale définissant un programme précis de codification prévu par l'annexe n'avait plus guère de sens.

${ }^{40}$ SILICANI (J.-L.), « La codification : instrument de réforme de l'Etat en France », précité, p. 641.

${ }^{41}$ Loi no ${ }^{\circ} 99-1071$ du 16 décembre 1999 portant habilitation du Gouvernement à procéder par ordonnances, à l'adoption de la partie législative de certains codes, J.O.R.F., 22 décembre 1999, p. 19040.
} 
L'article $1^{\mathrm{er}}$ précise la méthode de la codification puisque chaque code devra faire l'objet d'une ordonnance et que les dispositions codifiées seront celles en vigueur au moment de la publication de chaque ordonnance. Ce principe de codification à droit constant tolère trois exceptions. Les deux premières - respect de la hiérarchie des normes et cohérence rédactionnelle des textes rassemblés - constituent des ajustements classiques de la codification à droit constant. En revanche, la troisième - l'harmonisation du droit - est une nouveauté. Introduite par voie d'amendement par la Commission des lois du Sénat, elle permet à la codification de s'éloigner du droit constant pour procéder à une harmonisation nécessaire de l'état du droit et de résoudre ainsi les difficultés pouvant résulter du « rapprochement dans un code de plusieurs textes de sources différentes ${ }^{42}$. On précisera que cet objectif est expressément assigné à la codification par l'article 3 de la loi précitée relative aux droits des citoyens dans leurs relations avec les administrations.

Enfin l'article $1^{\text {er }}$ habilite le Gouvernement à étendre, éventuellement, l'application des dispositions codifiées à la Nouvelle-Calédonie, aux territoires d'outre-mer, à la collectivité territoriale de Saint-Pierre-et-Miquelon et à la collectivité territoriale de Mayotte. Compte tenu du principe de spécialité législative applicable aux territoires et collectivités d'outre-mer, la codification revêt un intérêt particulier. Dans son neuvième rapport annuel, la Commission supérieure de codification avait souligné l'importance de la codification qui permettait ainsi d'actualiser le droit applicable outre-mer et d'analyser, au cas par cas, la répartition des compétences entre l'Etat et la collectivité d'outre-mer ${ }^{43}$. Elle a également relevé la nécessité d'organiser une consultation préalable ${ }^{44}$ à l'extension des textes codifiés des collectivités et territoires intéressés. Dès lors, la loi d'habilitation, en autorisant l'extension des textes codifiés par les ordonnances, évite au Gouvernement le recours à des lois portant dispositions diverses, complexes et hétérogènes concernant l'outre-mer. Dès l'instant où la matière concernée relèvera de la compétence de l'Etat, les ordonnances assureront l'extension de ces dispositions à l'outre-mer en tenant compte des spécificités de chacune des collectivités ${ }^{45}$.

L'article 2 détermine, conformément à l'article 38 de la Constitution, le délai de publication des ordonnances et, pour chaque ordonnance, celui dans lequel doit intervenir le dépôt des projets de loi de ratification.

\footnotetext{
${ }^{42}$ Voir rapport de M. Alain Vidalies, au nom de la Commission des lois de l'Assemblée nationale, précité, p. 28.

${ }^{43}$ Commission supérieure de codification, Neuvième rapport annuel, op. cit., pp. 47 et ss. En raison de la complexité et de la diversité des régimes juridiques applicables à l'outre-mer, la Commission supérieure de codification a entrepris dans son rapport l'élaboration d'un guide méthodologique relatif à la codification des textes outre-mer.

${ }^{44}$ Ibid., pp. 48 et s.

${ }^{45}$ Le législateur a précisé l'extension éventuelle des dispositions codifiées à la collectivité territoriale de SaintPierre-et-Miquelon alors que le principe d'assimilation législative régit cette collectivité et que la loi y est applicable sans procédure d'extension. Mais avant la loi no 76-664 du 19 juillet 1976 relative à l'organisation de Saint-Pierre-et-Miquelon (J.O.R.F., 19-20 juillet 1976, p. 4323), cette collectivité était régie par le principe de spécialité législative. Dès lors, cette mention se justifie pour les textes antérieurs à l'intervention de cette loi et qui ne s'appliqueront que s'ils sont étendus expressément.
} 
Le premier délai tient compte de l'état d'avancement des différents codes et n'appelle pas de développements supplémentaires : six mois pour le Code rural, le Code de la santé publique et le Code de l'éducation; neuf mois pour le Code de commerce, le Code de l'environnement et le Code de justice administrative ; douze mois pour le Code de la route, le Code de l'action sociale et le Code monétaire et financier.

Le second délai a été modifié par le Sénat. Initialement le projet de loi d'habilitation prévoyait qu'un seul projet de ratification devait être déposé dans les quinze mois suivant la publication de la loi d'habilitation. Le Sénat a proposé le dépôt d'un projet de loi de ratification par ordonnance, dans les deux mois suivant la publication de l'ordonnance concernée, et au plus tard, pour les trois derniers codes, le dernier jour du quatorzième mois suivant la publication de la loi d'habilitation.

\section{II. - LE CONSEIL CONSTITUTIONNEL ET LA CODIFICATION : LA DECISION DU 16 DECEMBRE 1999}

A l'issue de l'adoption de la loi d'habilitation le 23 novembre 1999, les députés saisissent le Conseil constitutionnel d'un recours, suivi d'un mémoire complémentaire, en invoquant six séries de griefs ${ }^{47}$ auxquels la Haute juridiction répond dans la décision $\mathrm{n}^{\circ}$ 99421 DC du 16 décembre 1999. Toutefois, deux points essentiels sont à retenir : d'une part, cette décision déclare conforme à la Constitution la loi habilitant le Gouvernement à procéder à la codification par ordonnances et d'autre part, elle donne la qualification d' « objectif de valeur constitutionnelle » à l'accessibilité et à l'intelligibilité de la loi.

\section{A. - La loi d'habilitation est conforme à la Constitution}

La préoccupation principale des députés de l'opposition est la dépossession ${ }^{48} \mathrm{du}$ Parlement de ses prérogatives conférées par l'article 34 de la Constitution et une interprétation extensive par la loi d'habilitation de l'article 38. Mais il semble à la lecture de la saisine que l'hostilité des requérants porte moins sur la loi d'habilitation que sur la codification elle-

\footnotetext{
${ }^{46}$ C.C., décision $n^{\circ}$ 99-421 DC du 16 décembre 1999, Codification par ordonnances, J.O.R.F., 22 décembre 1999, p. 19041. A.J.D.A., 2000, p. 31, note J.-E. Schoettl ; MATHIEU (B.) et VERPEAUX (M.), « Chronique de jurisprudence constitutionnelle $\mathrm{n}^{\circ} 23$. Novembre 1999- Janvier 2000 (suite et fin) », L.P.A., 28 juillet 2000, $\mathrm{n}^{\circ}$ 150, p. 15 ; R.F.D.C., 41-2000, p.120, note D. Ribes ; R.T.D.C., janv.-mars 2000, p. 186, N. Molfessis.

${ }^{47}$ Le Conseil constitutionnel a dû se prononcer sur la violation des droits du Parlement, sur le respect des règles et principes à valeur constitutionnelle, sur la possibilité de procéder par ordonnances à l'élaboration des codes, sur le respect de la compétence organique du législateur, du pouvoir réglementaire et du domaine exclusif des lois de finances et des lois de financement de la sécurité sociale, sur les modalités de déclassement des dispositions de forme législative et de nature réglementaire et, enfin, sur le respect des articles 74 et 77 de la Constitution.

${ }^{48}$ La saisine a été précédée et certainement inspirée par la parution d'un article du Professeur Terré dénonçant le dessaisissement du Parlement : TERRE (F.), « Un Parlement démissionnaire », Le Figaro, 23 novembre 1999.
} 
même ${ }^{49}$. En tout état de cause, le Conseil constitutionnel répond aux griefs en faisant une stricte application des principes dégagés par sa jurisprudence à l'égard des ordonnances.

Les requérants estimaient que la loi déférée violait l'article 34 de la Constitution en ce que son domaine d'intervention était trop étendu, et que l'abrogation des dispositions existantes par le Gouvernement procédant à la codification des textes législatifs n'était pas prévue dans la loi d'habilitation. Ils prétendaient également que le recours à ce procédé ferait perdre leur caractère législatif aux dispositions codifiées par ordonnances.

Le Conseil constitutionnel, après avoir rappelé les termes de l'article 38, affirme que «le domaine de l'habilitation (...) peut comprendre toute matière qui relève du domaine de la loi en application de l'article $34 »$. Or la codification de textes législatifs appartient au pouvoir législatif, mais puisque l'article 38 permet précisément au Parlement d'autoriser le Gouvernement à légiférer par ordonnances, alors les codes relevant de ce domaine, peuvent être adoptés par ordonnances. En outre l'abrogation des dispositions législatives codifiées est liée au processus de codification, dès lors, précise le Conseil, la loi d'habilitation n'a pas à en faire mention. Néanmoins le juge constitutionnel ajoute que cette abrogation ne pourra avoir lieu qu'après avoir vérifié que les dispositions en cause dans le code ont été reprises ou implicitement abrogées, qu'elles présentaient une contrariété à la Constitution ou aux engagements internationaux ou enfin qu'elles relevaient du domaine réglementaire. Ces remarques ne sont pas inutiles car elles permettent de différencier la codification actuelle entreprise depuis 1989, des codifications anciennes qui ne supprimaient pas les textes codifiés. Ceux-ci demeuraient en vigueur et conservaient leur valeur. En cas de litige entre le code et le texte d'origine, c'est ce dernier qui était appliqué. Cette hypothèse ne peut plus exister avec l'affirmation constitutionnelle de l'abrogation des dispositions législatives codifiées $^{50}$.

En ce qui concerne la dernière crainte des requérants, il peut en effet sembler paradoxal que l'utilisation de l'article 38, en excluant le Parlement, conduise à donner valeur réglementaire - même momentanément - aux codes (notamment à la Partie législative du Code). Le Conseil ne s'en émeut nullement et relève simplement mais avec justesse que le régime contentieux des ordonnances « résulte directement de la combinaison des articles 38 et 61 de la Constitution ». En effet, il ne faudrait pas exagérer ${ }^{51}$ ce paradoxe car le Parlement est compétent en amont au moment du vote de la loi d'habilitation et en aval lorsqu'il doit procéder à la ratification des ordonnances, sur laquelle nous reviendrons. C'est également en invoquant cette solution que le Conseil évacue la méconnaissance du droit d'amendement par le Parlement évoquée par les requérants. Il ajoute une seconde possibilité pour les parlementaires d'utiliser leur droit d'amendement : déposer, à l'expiration du délai imparti au Gouvernement pour prendre des ordonnances, des propositions de loi visant à modifier ces ordonnances. Assurément ces possibilités demeurent pratiquement irréalisables en raison de

\footnotetext{
${ }^{49}$ Saisine du Conseil constitutionnel en date du 24 novembre 1999, J.O.R.F., 22 décembre 1999, pp. 19043 et ss.

${ }^{50}$ Voir « Codification. Entretien avec le Président Braibant », R.F.D.A., 2000, p. 494.

${ }^{51}$ Ibid., p. 495.
} 
l'absence de temps suffisant pour y procéder et de l'obstacle que constitue pour les propositions de loi l'inscription à l'ordre du jour ${ }^{52}$.

Les requérants invoquaient également une violation de l'article 38 de la Constitution qui impose au Gouvernement d'indiquer précisément la finalité et le champ matériel des mesures qu'il se propose de prendre par voie d'ordonnance ${ }^{53}$. De plus, dans l'exercice des pouvoirs conférés par l'article 38, le Gouvernement est tenu de respecter les règles et principes de valeur constitutionnelle ${ }^{54}$. Les requérants estimaient à titre principal que ce n'était pas le cas en l'espèce, que la loi aurait dû définir le contenu des codes et que la seule justification du retard dans le travail de codification était insuffisante.

Le Conseil constitutionnel rejette ces griefs. Il précise d'abord que l'urgence est « au nombre des justifications que le gouvernement peut invoquer pour recourir à l'article 38 de la Constitution». On notera que rien, dans les dispositions de l'article 38, ne laisse transparaître une idée d'urgence. Toutefois elle est une des justifications du recours à l'article 38 associée aux conditions juridiques nécessitées par ce même article ${ }^{55}$. Au surplus, le recours à l'article 38 pour accomplir la codification concourt à l'objectif de valeur constitutionnelle d'accessibilité et d'intelligibilité de la loi. Dès lors la justification est suffisante.

Par ailleurs, rappelons que le Gouvernement n'est pas tenu de faire connaître la teneur des ordonnances à prendre ${ }^{56}$. Dès lors l'imprécision du contenu des codes et de la notion de respect de la hiérarchie des normes soulevée par les requérants manque en fait. Le Conseil rejette ces griefs tout en précisant les termes de l'habilitation. Tout d'abord, la loi d'habilitation fixe le champ d'intervention des ordonnances (liste des matières législatives comprises dans neuf codes) et impose que la codification se fasse à droit constant. Cette dernière exigence implique que le Parlement connaît le contenu des futurs codes et qu'ainsi, il ne peut pas s'estimer dépossédé de son pouvoir de légiférer ${ }^{57}$. Le premier grief est neutralisé. Quant au second, le Conseil fait référence à la hiérarchie des normes à propos de la «supériorité du traité sur la loi ». C'est la première fois qu'apparaît dans une décision cette expression depuis longtemps consacrée en doctrine. La pyramide kelsénienne pénètre donc officiellement le droit français. Par ailleurs, alors que la saisine ne faisait pas allusion à la possibilité laissée aux dispositions codifiées d'harmoniser l'état du droit, le Conseil indique que «les modifications rendues nécessaires pour "harmoniser l'état du droit » doivent se

\footnotetext{
${ }^{52}$ MATHIEU (B.) et VERPEAUX (M.), « Chronique de jurisprudence constitutionnelle $\mathrm{n}^{\circ} 23$. Novembre 1999Janvier 2000 (suite et fin) », L.P.A., 28 juillet 2000, $\mathrm{n}^{\circ} 150$, p. 16.

${ }^{53}$ C.C., décision n ${ }^{\circ}$ 76-72 DC du 12 janvier 1977, Territoires des Afars et des Issas, R.J.C., I-42 ; C.C., décision $\mathrm{n}^{\circ}$ 86-207 des 25 et 26 juin 1986, Privatisations, R.J.C., I-254, cons.13 ; C.C., décision n ${ }^{\circ} 86-208$ DC des $1^{\text {er }}$ et 2 juillet 1986, Découpage électoral I, R.J.C., I-262, cons.18 et 27 ; C.C., décision n 95-370 DC du 30 décembre 1995, R.J.C., I-650, cons. 15 à 17.

${ }^{54}$ C.C., décision $\mathrm{n}^{\circ}$ 81-134 DC du 5 janvier 1982, Loi d'orientation sociale, R.J.C., I-113, cons. 6 ; C.C., décision n ${ }^{\circ} 86-207$ des 25 et 26 juin 1986, Privatisations, précitée, cons. 14 et 15.

${ }^{55}$ BOYER-MERENTIER (C.), Les ordonnances de l'article 38 de la Constitution du 4 octobre 1958, Paris, Economica, Aix-en-Provence, P.U.A.M., 1996, pp. 30 et ss.

${ }^{56}$ C.C., décision n ${ }^{\circ} 86-207$ DC des 25 et 26 juin 1986, Privatisations, précitée, cons. 13 et 21.

${ }^{57}$ Voir MATHIEU (B.) et VERPEAUX (M.), «Chronique de jurisprudence constitutionnelle ${ }^{\circ} 23$. Novembre 1999- Janvier 2000 (suite et fin) », précité, pp. 16 et s.
} 
borner à remédier aux incompatibilités pouvant apparaître entre des dispositions soumises à codification ». Il s'appuie sur les travaux préparatoires dont il ressort que cette formule strictement interprétée doit seulement permettre aux ordonnances «de remédier à des incompatibilités patentes entre dispositions à codifier, dans le sens le plus conforme aux exigences constitutionnelles, à l'économie générale d'un texte ou à l'évolution générale de la législation $\gg^{58}$.

Enfin, le Conseil constitutionnel rappelle l'interdiction d'utiliser l'article 38 dans le domaine des lois de finances ou dans celui, depuis la révision constitutionnelle du 22 février 1996, des lois de financement de la sécurité sociale.

\section{B. - Le principe d'accessibilité et d'intelligibilité de la loi : objectif de valeur constitutionnelle}

Parmi les griefs relevés, les requérants considéraient comme insuffisante la justification du recours à la procédure de l'article 38 reposant sur l'urgence et par là, contestaient la nécessité même de la codification. Après avoir souligné que l'achèvement des codes est lié à la satisfaction de l'intérêt général, le Conseil constitutionnel ajoute incidemment que le processus de codification « répond au demeurant à l'objectif de valeur constitutionnelle d'accessibilité et d'intelligibilité de la loi ». Il fonde cette nouvelle norme constitutionnelle sur les articles 4, 5, 6 et 16 de la Déclaration des droits de l'homme et du citoyen. Il indique en effet que "l'égalité devant la loi, énoncée par l'article 6 de la Déclaration des droits de l'homme et du citoyen, et «la garantie des droits» requise par son article 16 pourraient ne pas être effectives si les citoyens ne disposaient d'une connaissance suffisante des normes qui leur sont applicables; qu'une telle connaissance est en outre nécessaire à l'exercice des droits et libertés garantis tant par l'article 4 de la Déclaration, en vertu duquel cet exercice n'a de bornes que celles déterminées par la loi, que par son article 5, aux termes duquel «tout ce qui n'est pas défendu par la loi ne peut être empêché, et nul ne peut être contraint à faire ce qu'elle n'ordonne pas »».

Alors qu'il n'était pas contraint de viser un principe, le Conseil érige au rang des objectifs de valeur constitutionnelle l'exigence d'accessibilité et d'intelligibilité de la loi. Dégagé des contingences de fond, ce principe constitue un apport original non seulement quant à la qualification d' «objectif de valeur constitutionnelle » mais également quant à sa signification.

- L'objectif de valeur constitutionnelle :

La notion d' " objectif de valeur constitutionnelle » est apparue dans la jurisprudence dès $1982^{59}$ et a été depuis régulièrement utilisée dans différents secteurs de la vie publique

\footnotetext{
${ }^{58}$ SCHOETTL (J.-E.), «Codification par ordonnances», A.J.D.A., 2000, p. 35.

${ }^{59}$ C.C., décision $\mathrm{n}^{\circ}$ 82-141 DC du 27 juillet 1982, Communication audiovisuelle, R.J.C. I-126 : objectifs de la

« liberté d'autrui » et de « la préservation du caractère pluraliste des courants socio-culturels ».
} 
nationale: le pluralisme ${ }^{60}$, la protection de la santé publique ${ }^{61}$, la sauvegarde de l'ordre public $^{62}$, la liberté d'autrui ${ }^{63}$, la lutte contre la fraude fiscale ${ }^{64}$, la possibilité de disposer d'un logement décent ${ }^{65}$ (la liste des objectifs ainsi que les décisions qui les illustrent ne sont pas exhaustives). Cette expression illustre l'enrichissement des méthodes du juge constitutionnel face à l'évolution du contexte politique et social. Qu'il s'agisse de la formation, de la fonction ou de la place des objectifs de valeur constitutionnelle au sein de la hiérarchie des normes, on souscrit à l'étude de Bertrand Faure ${ }^{66}$ qui tente, avec succès, de dégager les caractéristiques de ces normes contemporaines et en propose une définition unitaire : «les objectifs de valeur constitutionnelle se ramènent donc à un procédé juridique posant en termes d'exigence de rang constitutionnel une éthique sociale limitative des libertés individuelles sous le contrôle directif du Conseil constitutionnel ${ }^{67}$.

De rang constitutionnel, donc supérieurs aux lois, les objectifs de valeur constitutionnelle s'appuient sur des éléments du bloc de constitutionnalité et font office de norme de conciliation. Dès lors il s'agit pour le juge constitutionnel de trouver le meilleur compromis possible entre l'affirmation d'une liberté et la défense d'intérêts matérialisés par l'objectif. Cette fonction signifie qu'aucun principe de valeur constitutionnelle ne revêt un caractère absolu et est toujours susceptible d'être concilié avec d'autres principes de valeur constitutionnelle $^{68}$, donc d'être limité ${ }^{69}$. Toutefois la poursuite d'un objectif de valeur constitutionnelle ne doit pas permettre d'écarter le mécanisme du « cliquet anti-retour ${ }^{70}$. Ainsi une loi qui met en œuvre un objectif de valeur constitutionnelle ne pourra être abrogée sans que soient accordées des garanties équivalentes.

Le contenu de cette catégorie est variable (voir supra) mais les objectifs consacrés par la jurisprudence constitutionnelle ont tous eu, jusqu'à présent, pour vocation de restreindre l'exercice des droits fondamentaux afin de privilégier l'intérêt général : «le perfectionnement de l'ordre social est leur manteau commun. L'individu y est reçu dans ses relations avec les autres individus mais toujours pour l'intérêt de la paix publique sous l'encadrement des valeurs portées par ces objectifs (logement décent pour tous, ordre, pluralisme, santé, lutte

\footnotetext{
${ }^{60}$ C.C., décision n ${ }^{\circ}$ 84-181 DC des 10 et 11 octobre 1984, Entreprises de presse, R.J.C., I-199: objectifs de «pluralisme des courants d'expression socio-culturels» et de «transparence financière des entreprises de presse ».

${ }^{61}$ C.C., décision n ${ }^{\circ}$ 89-269 DC du 22 janvier 1990, Egalité entre français et étrangers, R.J.C., I-392.

${ }^{62}$ C.C., décision n ${ }^{\circ} 82-141$ DC du 27 juillet 1982, précitée.

${ }^{63}$ Ibid.

${ }^{64}$ C.C., décision n ${ }^{\circ}$ 86-209 DC du 3 juillet 1986, Loi de finances rectificative pour 1986, R.J.C., I-266.

${ }^{65}$ C.C., décision n ${ }^{\circ}$ 94-359 DC du 15 janvier 1995, Diversité de l'habitat, R.J.C., I-630.

${ }^{66}$ FAURE (B.), «Les objectifs de valeur constitutionnelle : une nouvelle catégorie juridique ? », R.F.D.C., 211995, pp. 47-77.

${ }^{67}$ Ibid., p. 77.

${ }^{68}$ Voir FAVOREU (L.), PHILIP (L.), Les grandes décisions du Conseil constitutionnel, Paris, Dalloz, 10è éd., 1999, p. 611.

${ }^{69}$ Voir en ce sens DRAGO (G.), Contentieux constitutionnel français, Paris, P.U.F., Coll. Thémis, 1998, p. 237 ; LUCHAIRE (F.), Le Conseil constitutionnel, Tome II - Jurisprudence, Première partie : L'individu, Paris, Economica, 2è éd., 1998, pp. 146 et s.

${ }^{70}$ Pour une première manifestation de la jurisprudence dite du «cliquet »: C.C., décision n $83-165$ DC du 20 janvier 1984, Libertés universitaires, R.J.C., I-171.
} 
contre la fraude...). Ceux-ci sont plus subtilement tournés vers la supériorité de l'intérêt général sur la personne $»^{71}$.

- Le sens du nouvel objectif :

En ce qui concerne sa signification le nouvel objectif d'accessibilité et d'intelligibilité de la loi répond aux critères précédemment dégagés mais s'en distingue par son originalité. Cet objectif s'appuie effectivement sur une source écrite appartenant au bloc de constitutionnalité : la Déclaration des droits de l'homme et du citoyen et sert l'intérêt général dans la mesure où, s'adressant aux pouvoirs publics, obligation leur est faite de rendre la loi et le droit intellectuellement compréhensible et matériellement accessible.

Mais la fonction de conciliation est inexistante dans cette décision. Le Conseil constitutionnel affirme un nouvel objectif sans l'appliquer immédiatement au cas présent. Et pour cause, les requérants n'avaient soulevé aucune atteinte à un tel principe, l'objectif venant seulement en appui de la constitutionnalité déjà conférée à la loi par l'article 38 de la Constitution. L'objectif recouvrera sa fonction originelle dans des cas ultérieurs mais il ne le fait pas en l'espèce.

En poussant à l'extrême sa portée, certains ont ainsi pu affirmer que cette décision proposait une conception systémique du droit: "Pour quoi est fait le système juridique ? Il est l'organisation grâce à laquelle la personne peut jouer dans le système politique son rôle de citoyen (...) Pour que la citoyenneté soit effective, il faut que la personne puisse obtenir la garantie de ses droits. Pour que la garantie de ses droits soit effective, il faut que la loi lui soit accessible. Pour que la loi soit accessible à la personne, il faut qu'elle lui soit intelligible $»^{72}$.

Cette interprétation porteuse de nombreuses potentialités est peut-être excessive et, sans hypothéquer les utilisations futures de l'objectif par le Conseil constitutionnel, on retiendra cependant que cette décision est le corollaire de l'adage «Nul n'est censé ignorer la $l o i \gg^{73}$ qui s'adresse aux individus. Cette maxime, au caractère sans nul doute fictif ${ }^{74}$, oblige l'individu à connaître le droit préalablement à son application effective. Il est cependant acquis qu'actuellement "la vérité de la maxime procède de ce que la force obligatoire de la loi ne tient ni à sa connaissance ni à sa méconnaissance, mais simplement à ce qu'elle est l'acte du pouvoir souverain $\gg^{75}$. Cet adage demeure seulement une règle de fond organisant l'irrecevabilité d'un argument et donc une règle technique sans lien avec le sens commun. Ceci étant, l'objectif d'accessibilité et d'intelligibilité de la loi est finalisé et pose une obligation de résultat à l'encontre de l'Etat. Désormais, les pouvoirs publics ont le devoir

\footnotetext{
${ }^{71}$ FAURE (B.), «Les objectifs de valeur constitutionnelle : une nouvelle catégorie juridique ? », précité, p. 59.

${ }^{72}$ FRISON-ROCHE (M.-A.) et BARANES (W.), «Le principe constitutionnel de l'accessibilité et de l'intelligibilité de la loi », D., Doctrine, 2000, p. 363.

${ }^{73}$ Voir en ce sens RIBES (D.), «Codes en stock. Le Conseil constitutionnel et les aléas de la codification », R.F.D.C., 41-2000, pp. 121 et s.

${ }^{74}$ GUILLIEN (R.), «Nul n'est censé ignorer la loi », in Mélanges Roubier, T. 1, 1961, p. 253 ; TERRE (F.), «Le rôle actuel de la maxime Nul n'est censé ignorer la loi », Travaux et recherches de l'Institut de droit comparé, T. XXX, 1966, pp. 91 et ss ; NICOLAU (G.), «"Inaccessible droit !" », R.R.J., 1998-1, pp. 15-49.

${ }^{75}$ ROLAND (H.), BOYER (L.), Adages du droit français, Paris, Litec, 4è éd., 1999, p. 584.
} 
d'organiser l'accès matériel et intellectuel du droit aux individus. Cette solution généralise un principe déjà échafaudé dans des décisions précédentes du juge constitutionnel relatives à la loi d'orientation et d'incitation sur la réduction du temps de travail ${ }^{76}$ et au $\operatorname{PACS}^{77}$.

Même s'il n'est pas opérationnel en l'espèce, ce nouvel objectif de valeur constitutionnelle enrichit incontestablement le droit constitutionnel.

Il constitue tout d'abord une nouvelle facette du principe de sécurité juridique. Consacré en droit allemand et en droit communautaire, le juge constitutionnel français refuse de l'ériger en principe de valeur constitutionnelle. Mais paradoxalement le Conseil n'hésite pas à reconnaître valeur constitutionnelle «aux exigences qui en constituent la substance ${ }^{78}$. Ainsi invoque-t-il les exigences constitutionnelles de garantie des droits, de sûreté, et de liberté contractuelle afin de poser des limites constitutionnelles respectivement aux validations législatives ${ }^{79}$, à la non-rétroactivité des lois ${ }^{80}$, aux atteintes portées à l'économie des conventions et contrats en cours ${ }^{81}$. Il a reconnu par ailleurs le principe de clarté de la $1 \mathrm{oi}^{82}$ sur le fondement de l'article 34 de la Constitution et celui de stabilité juridique ${ }^{83}$.

La présente décision ne reconnaît pas valeur constitutionnelle au principe de sécurité juridique ${ }^{84}$ mais en révèle une nouvelle composante : l'accessibilité et l'intelligibilité de la loi. Le Conseil constitutionnel affine sa ligne jurisprudentielle, enracine la sécurité juridique dans l'ordre constitutionnel mais ne franchit pas le Rubicon. Malgré la large faveur dont ce principe jouit, il est actuellement peu probable que le Conseil lui attribue valeur constitutionnelle. Les raisons en sont connues. En premier lieu il n'existe aucune formulation explicite de la sécurité juridique au sein d'un texte appartenant au bloc de constitutionnalité et l'on sait combien le juge constitutionnel répugne à dégager un principe constitutionnel sans attache textuelle expresse. Sans doute la référence à l'article 16 de la Déclaration a pu être perçue comme fondant l'exigence de sécurité juridique ${ }^{85}$ mais c'est insuffisant. En second lieu la sécurité juridique ne possède aucun contour clairement dessiné et la reconnaissance

\footnotetext{
${ }^{76}$ C.C., décision n ${ }^{\circ}$ 98-401 DC du 10 juin 1998, Loi sur les 35 heures, J.O.R.F., 14 juin 1998, p. 9033.

${ }^{77}$ C.C., décision n ${ }^{\circ}$ 99-419 DC du 9 novembre 1999, Pacte civil de solidarité, J.O.R.F., 16 novembre 1999, p. 16962: il semble que cette décision impose à toute loi affectant des droits ou des obligations de prévoir l'information des personnes concernées.

${ }^{78}$ MATHIEU (B.), «La sécurité juridique : un principe constitutionnel clandestin mais efficient » in Mélanges Gélard, Paris, Montchrestien, 1999, p. 302.

${ }^{79}$ C.C., décision $\mathrm{n}^{\circ}$ 93-335 DC du 21 janvier 1994, Urbanisme et construction, R.J.C., I-576 ; C.C., décision $\mathrm{n}^{\circ}$ 97-390 du 19 novembre 1997, Fiscalité applicable en Polynésie française, R.J.C., I-717.

${ }^{80}$ C.C., $\mathrm{n}^{\circ}$ 91-298 DC du 24 juillet 1991, Dispositions fiscales rétroactives, R.J.C., I-465.

${ }^{81}$ C.C., décision n ${ }^{\circ}$ 98-401 DC du 10 juin 1998, Loi sur les 35 heures, précitée ; C.C., décision n ${ }^{\circ}$ 99-416 DC du 28 juillet 1999, J.O.R.F., 28 juillet 1999, p. 11250.

${ }^{82}$ C.C., décision n ${ }^{\circ}$ 98-401 DC du 10 juin 1998, Loi sur les 35 heures, précitée ; C.C., décision n 99-407 DC du 14 janvier 1999, J.O.R.F., 24 janvier 1999, p. 4238.

${ }^{83}$ C.C., décision n ${ }^{\circ}$ 93-335 DC du 21 janvier 1994, précitée.

${ }^{84}$ Contra MOLFESSIS (N.), «Les illusions de la codification à droit constant et la sécurité juridique », R.T.D.C., janv.-mars 2000, p. 188 ; c'est avec plus de nuance que MM. Mathieu et Verpeaux affirment la reconnaissance du principe de sécurité juridique dans cette décision puisqu'ils la qualifient d' «implicite»: MATHIEU (B.) et VERPEAUX (M.), « Chronique de jurisprudence constitutionnelle ${ }^{\circ} 23$. Novembre 1999Janvier 2000 (suite et fin) », précité, p. 17.

${ }^{85}$ Voir en ce sens MATHIEU (B.), «La sécurité juridique : un produit d'importation dorénavant "made in France" », D., 2000, n ${ }^{\circ}$, Point de vue, VII.
} 
ponctuelle de multiples facettes de ce principe dans la jurisprudence constitutionnelle accentue l'indétermination des limites à sa définition et contribue ainsi à renforcer l'idée d'une construction polysémique de la sécurité juridique.

Le second apport du nouvel objectif d'accessibilité et d'intelligibilité de la loi est la consécration de la codification. Cette dernière est le moyen, mais pas l'unique, d'atteindre le but énoncé dans la décision : le devoir de l'Etat est de permettre au citoyen de comprendre et d'accéder à la loi qui lui est applicable. La codification devient un instrument de prédilection pour l'Etat qui doit rendre la loi matériellement et intellectuellement accessible. En liant ces deux aspects à celui de l'effectivité des droits et libertés, le Conseil constitutionnel accentue l'obligation imposée à l'Etat. Désormais chacun doit pouvoir accéder à la loi pour que ses droits et libertés soient effectifs.

C'est un raisonnement identique qui a conduit le Conseil constitutionnel, sur le fondement de l'article 16 de la Déclaration des droits de l'homme et du citoyen, à consacrer et constitutionnaliser le droit d'agir en justice ${ }^{86}$. Pour que ses droits soient effectifs le justiciable devait pouvoir se plaindre devant un juge en cas de violation. Dans le cas présent l'effectivité des droits et libertés est réalisable parce que l'Etat a l'obligation de mettre à la disposition du citoyen les moyens nécessaires à l'accessibilité et à l'intelligibilité de la loi. La codification est l'un de ces moyens. Les glorificateurs du processus de codification seront confortés par cette décision qui donne un fondement constitutionnel à la codification et les sceptiques continueront à s'interroger sur ses imperfections. Quoiqu'il en soit, la décision 421 DC apporte sa pierre à la consolidation de l'Etat de droit.

\section{III. - LA CONSOLIDATION DE L'ETAT DE DROIT}

L'Etat de droit, par opposition à l'Etat de police, est la qualification d'un régime démocratique et libéral «dans lequel la toute puissance du pouvoir trouve sa limite dans la règle juridique qu'il est tenu de respecter ${ }^{87}$ et a pour finalité la protection du citoyen contre l'arbitraire. La recherche perpétuelle d'un Etat de droit sans cesse amélioré conduit les pouvoirs publics à renouveler les méthodes utilisées. La codification semble devenir une de ces méthodes de consolidation.

En effet pour atteindre un tel dessein, le droit positif doit être connu et compréhensible ou du moins s'efforcer de l'être. Or la prolifération, l'instabilité et même parfois le flou de la règle sont autant d'obstacles à sa réalisation. La codification peut être un des remèdes permettant le retour à la stabilité. Mieux encore. La décision 421 DC est l'affirmation constitutionnelle selon laquelle la codification, non seulement est l'une des justifications du recours à l'article 38 de la Constitution, mais surtout, poursuit l'objectif de valeur

\footnotetext{
${ }^{86}$ C.C., décision n ${ }^{\circ} 96-373$ du 9 avril 1996, Autonomie de la Polynésie française I, R.J.C., I-660.

${ }^{87}$ RIVERO (J.), «Etat de droit, état du droit », in Mélanges Braibant, L’Etat de droit, Paris, Dalloz, 1996, p. 609.
} 
constitutionnelle d'accessibilité et d'intelligibilité de la loi. Le Conseil constitutionnel, dans cette décision satisfait une des exigences de l'Etat de droit : rendre le droit accessible à ceux qu'il concerne.

Toutefois, affermir 1'Etat de droit nécessite une vigilance de tous les instants. En particulier, les efforts accomplis par le gouvernement, le législateur et le Conseil constitutionnel dans la poursuite du processus de codification ne doivent pas fléchir comme le souligne la Commission supérieure de codification dans son rapport d'activité pour l'année $2000^{88}$.

\section{A. - La codification : un moyen de rendre le droit accessible}

Etant donné le retard pris dans le processus de codification, le procédé choisi afin d'accélérer cette entreprise fut de recourir aux ordonnances. Cette préférence a suscité de nombreuses craintes que les travaux préparatoires et la décision du Conseil constitutionnel relatifs à la loi d'habilitation ont pu dissiper.

La première critique, précédemment évoquée, est celle relative à la justification du recours aux ordonnances : l'urgence. Sans doute, le Gouvernement qui l'invoque est-il luimême à l'origine du retard dans le travail de codification lié à l'encombrement parlementaire puisque, selon l'article 48 de la Constitution, il est maître de l'ordre du jour parlementaire ${ }^{89}$. Mais cette justification satisfait le Conseil. $\mathrm{Si}$, avant la décision le processus de codification était une nécessité, il est d'autant plus avec la décision 421 DC dans la mesure où la codification sert un objectif de valeur constitutionnelle. Il faut en outre reconnaître que face aux besoins requis par la politique gouvernementale en matière économique ou sociale, la codification n'attire pas préférentiellement l'ordre du jour des assemblées.

Il est également reproché à la décision du Conseil de laisser le champ libre au Gouvernement pour s'affranchir du Parlement et piétiner sa compétence. S'il est vrai que l'habilitation est large, il n'en demeure pas moins qu'elle est encadrée et respectueuse de l'article 38 et des principes dégagés par le Conseil constitutionnel en la matière ${ }^{90}$. En outre, il ne s'agit pas de confier aux ordonnances le soin de modifier l'état du droit comme cela a pu être le cas dans des domaines aussi importants que les privatisations, le découpage des circonscriptions électorales ou la sécurité sociale, il s'agit seulement de mettre en ordre des textes existants. C'est le principe de la codification à droit constant que le Conseil ne manque

\footnotetext{
${ }^{88}$ Commission supérieure de codification, Onzième rapport annuel (2000), http://www.ladocfrancaise.gouv.fr. Voir ORLOWSKA (M.), «L'année 2000, une étape décisive pour la codification », L.P.A., $1^{\mathrm{er}}$ août 2001, $\mathrm{n}^{\circ}$ 152 , p. 4.

${ }^{89}$ Voir en ce sens MOLFESSIS (N.), «Les illusions de la codification à droit constant et la sécurité juridique », précité, p. 187.

${ }^{90}$ Selon les décisions $\mathrm{n}^{\circ}$ 86-207 DC et $\mathrm{n}^{\circ}$ 86-208 DC précitées, la loi d'habilitation doit préciser le domaine d'intervention des mesures envisagées ; selon la décision n ${ }^{\circ}$ 76-72 DC, précitée, le Gouvernement doit indiquer la finalité des mesures à prendre ; selon les décisions n 81-134 DC et nº 86-207 DC, précitées, le Gouvernement n'est pas dispensé du respect des principes constitutionnels dans le cadre des pouvoirs que lui confère l'article 38.
} 
pas de rappeler: celui- ci «s'oppose à ce que soit réalisée une modification du fond des matières législatives codifiées ». L'utilisation des ordonnances pour adopter des réformes est, semble-t-il, tout à fait différente de l'utilisation des ordonnances pour réorganiser l'ordonnancement juridique.

Nous avons vu précédemment que la codification à droit constant admet des aménagements parmi lesquels l'harmonisation de l'état du droit. Cette formule aurait pu être sujette à caution. Mais les travaux préparatoires ${ }^{91}$ dissipent les interrogations en indiquant que les ordonnances devront se limiter aux incompatibilités apparaissant entre des dispositions soumises à codification. C'est le cas, par exemple du Code pénal et du Code de la route qui contiennent tous deux des infractions définies en termes identiques mais punies différemment. Cette formule est strictement interprétée et donc limitée dans ses possibilités ${ }^{92}$. C'est ce que semble confirmer le Conseil constitutionnel lorsqu'il précise son sens en s'appuyant sur les travaux préparatoires alors que la saisine n'en faisait pas mention (voir supra).

Ces difficultés ne doivent cependant pas occulter l'apport de la décision 421 DC qui, au-delà des inconvénients inhérents à toutes les ordonnances, consacre, sans pour autant acquérir valeur constitutionnelle, la codification comme un moyen de faciliter l'accessibilité et l'intelligibilité de la loi.

On constate ainsi que, progressivement, se dessine la mise en place d'un système tourné tout entier vers la consolidation formelle de l'Etat de droit. Jusqu'à présent le Conseil constitutionnel a développé, pour une majeure partie, une jurisprudence ayant pour finalité de reconnaître et protéger les droits et libertés fondamentaux, édifiant ainsi un véritable droit constitutionnel des libertés. Il s'attache désormais à rendre le droit et ses institutions accessibles aux citoyens. C'est par une démarche pragmatique que, progressivement, il se dote de principes de rang constitutionnel (droit d'agir en justice, accessibilité et intelligibilité de la loi) à réaliser ou à concilier et des instruments pour y parvenir (la codification), afin d'aboutir à la finalité suprême, l'effectivité des droits et libertés qui est une des exigences de l'Etat de droit qui, désormais «entre pleinement dans la réalité vécue par les hommes ${ }^{93}$.

La mise en œuvre de la réforme de l'Etat et des services publics ${ }^{94}$ est un contexte privilégié pour le législateur et le Gouvernement de participer à cette entreprise. Déjà un effort a été accompli pour organiser le libre accès à l'information administrative ${ }^{95}$ et la loi $n^{\circ}$

\footnotetext{
${ }^{91}$ Rapport de M. Alain Vidalies, au nom de la Commission des lois de l'Assemblée nationale, précité, p. 28.

${ }^{92}$ Contra MOLFESSIS (N.), «Les illusions de la codification à droit constant et la sécurité juridique », précité, pp. 191 et ss. ; notamment p. 193 : «La loi reconnaît désormais expressément aux codificateurs la faculté de modifier substantiellement les textes existants en vue de permettre "l'harmonisation du droit» ». M. Molfessis interprète largement cette disposition et tire des conséquences néfastes pour la sécurité juridique tout en ignorant les travaux préparatoires et la décision du Conseil constitutionnel afin de développer une critique de la codification par ordonnances voire de la codification tout court.

${ }^{93}$ RIVERO (J.), «Etat de droit, état du droit », précité, p. 614.

${ }^{94}$ Circulaire du 26 juillet 1995 du Premier Ministre relative à la préparation et à la mise en œuvre de la réforme de l'Etat et des services publics, précitée, p. 11217.

${ }_{95}$ Circulaire du 17 décembre 1998 relative à la diffusion des données juridiques sur les sites Internet des administrations, J.O.R.F., 24 décembre 1998, p. 19487.
} 
2000-231 relative aux droits des citoyens dans leurs relations avec les administrations ${ }^{96}$ institue dans son article 2 l'obligation pour les autorités administratives d'organiser un accès simple aux règles de droit qu'elles édictent et fait de la mise à disposition et de la diffusion des textes juridiques une mission de service public. Selon l'article 3 l'accomplissement de cette mission appartient à la codification.

Ce processus, auquel participent activement le Parlement et le Conseil constitutionnel, reflète la situation actuelle dans laquelle la codification, après maintes tribulations, occupe une place de choix. Désormais l'état du droit est indissociable de l'Etat de droit.

Mais il ne suffit pas de s'attendrir sur le parcours déjà réalisé, encore faut-il redoubler d'efforts pour améliorer et « cultiver » la codification afin de consolider l'Etat de droit.

\section{B. - La codification : une poursuite sans relâche}

Le respect des délais fixés par la loi d'habilitation ${ }^{97}$ et la publication des neuf codes au cours de l'année 2000 constituent une grande satisfaction mais le processus de codification, dans toutes ses phases, reste sujet à de nombreuses faiblesses auxquelles il conviendra de remédier. Le onzième rapport de la Commission supérieure de codification pour l'année 2000 dresse le bilan du travail accompli et met en évidence les difficultés rencontrées tout en traçant les voies pour l'avenir.

Tout d'abord, le recours à la procédure de l'article 38 laisse entière la question de la ratification des ordonnances. En effet si le projet de loi d'habilitation prévoit le délai de dépôt du projet de loi de ratification, il ne prévoit aucun délai au cours duquel les ordonnances devront être ratifiées. Cette absence est préjudiciable à la stabilité juridique puisque pendant une durée indéterminée les ordonnances non ratifiées ont un caractère réglementaire ${ }^{98}$. Dès lors, les codes non ratifiés n'auront qu'une valeur réglementaire et pourront être annulés par la voie du recours pour excès de pouvoir devant le Conseil d'Etat.

C'est pourquoi, le projet de loi d'habilitation, sur proposition de la Commission des lois du Sénat ${ }^{99}$, prévoit le dépôt non d'un seul projet de ratification mais de plusieurs, chacun de ces projets doit correspondre à une ordonnance et être déposé dans le délai de deux mois à compter de la publication de l'ordonnance. Cette initiative prévient les risques d'instabilité

\footnotetext{
${ }^{96}$ Précitée.

${ }^{97}$ Code rural : ordonnance $n^{\circ}$ 2000-550 du 15 juin 2000, Code de l'éducation : ordonnance $n^{\circ} 2000-549$ du 15 juin 2000, Code de la santé publique : ordonnance $\mathrm{n}^{\circ} 2000-548 \mathrm{du} 15$ juin 2000, Code de commerce : ordonnance $\mathrm{n}^{\circ}$ 2000-912 du 18 septembre 2000, Code de l'environnement : ordonnance $\mathrm{n}^{\circ} 2000-914$ du 18 septembre 2000, Code de la justice administrative : ordonnance $\mathrm{n}^{\circ} 2000-387 \mathrm{du} 4$ mai 2000, Code de la route : ordonnance $\mathrm{n}^{\circ} 2000-930 \mathrm{du} 22$ septembre 2000 (et ordonnance $\mathrm{n}^{\circ} 2000-1255$ du 21 décembre 2000 modifiant certaines dispositions annexées à la précédente ordonnance), Code de l'action sociale et des familles :

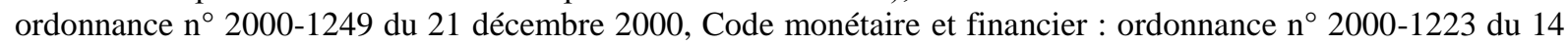
décembre 2000.

${ }^{98}$ C.E., Sect., 3 novembre 1961, Damiani, rec. Lebon, p. 607.

${ }^{99}$ Rapport de M. Patrice Gélard, au nom de la Commission des lois du Sénat, précité, p. 15 et 25 : le rapporteur souligne les risques d'insécurité juridique résultant d'une absence de ratification.
} 
sans toutefois éliminer l'insécurité juridique. La seule issue est d'exhorter le Gouvernement à inscrire à l'ordre du jour des assemblées, dans les plus brefs délais, les projets de loi de ratification afin de procéder à la ratification explicite des ordonnances. Cette solution serait la plus satisfaisante mais s'il est souhaitable que le Parlement examine au fond les codes qui lui seront soumis pour une ratification explicite, on peut douter qu'il ait le temps de vérifier tous les articles de tous les codes et d'exercer son droit d'amendement comme le prévoit la décision 421 DC. Toutefois on soulignera que les codes reprennent des dispositions existantes précédemment adoptées par le Parlement, ce qui minimise l'absence d'examen minutieux par ce dernier du contenu de chaque ordonnance ${ }^{100}$. Si la ratification explicite est inexistante, l'incertitude liée aux ratifications implicites ${ }^{101}$, impliquées ${ }^{102}$ ou par validation ${ }^{103}$, augmentera l'insécurité juridique et méconnaîtra ainsi le nouvel objectif de valeur constitutionnelle dégagé par le Conseil. En effet, la complexité du régime actuel des ordonnances accentuerait d'une part l'incompréhension des textes codifiés par ordonnances et d'autre part, les difficultés d'interprétation liées à leur valeur normative ${ }^{104}$.

Ensuite, en ce qui concerne l'entrée en vigueur des codes, les usagers doivent être préparés aux nouveaux changements introduits. Cette acclimatation pratique ne s'effectuera correctement que si, en premier lieu, les tables de concordance accompagnant les dispositions nouvelles sont publiées au Journal officiel en même temps que le nouveau code. Outil indispensable à tous les praticiens du droit, elles participent également à l'accessibilité du droit. Or jusqu'à présent, la simultanéité entre leur publication et celle d'un nouveau code a fait défaut ${ }^{105}$.

En second lieu l'adaptation des usagers se réalisera d'autant mieux si l'entrée en vigueur des codes est différée. C'est la leçon qu'il faut tirer de l'entrée en vigueur immédiate du code de commerce qui a conduit à de nombreux désagréments. En effet, au moment même où le code de commerce entrait en vigueur, sans information préalable concernant les nouvelles numérotation et organisation de ce code, «des engagements, des contrats, des jugements fondés sur des anciennes dispositions étaient en cours d'élaboration ${ }^{106}$. Le gain

\footnotetext{
${ }^{100}$ Voir en ce sens « Codification. Entretien avec le Président Braibant », précité p. 495.

101 C.C., décision $\mathrm{n}^{\circ} 72-73$ L du 29 février 1972, Participation des salariés, R.J.C., II-48 : la ratification implicite doit résulter «d'une manifestation implicitement mais clairement exprimée par le Parlement ».

${ }^{102}$ C.C., décision n ${ }^{\circ}$ 86-224 DC du 23 janvier 1987, Conseil de la concurrence, R.J.C., I-303. : la ratification de tout ou partie des dispositions d'une ordonnance visée par l'article 38 de la Constitution peut résulter d'une loi qui, « sans avoir cette ratification pour objet direct, l'implique nécessairement ».

${ }^{103}$ C'est le cas de la loi $\mathrm{n}^{\circ}$ 96-1093 du 16 décembre 1996 relative à l'emploi dans la fonction publique et à diverses mesures d'ordre statutaire (J.O.R.F., 17 décembre 1996, p. 18512) qui valide les dispositions du Titre IV de l'ordonnance $\mathrm{n}^{\circ}$ 96-346 du 24 avril 1996 portant réforme de l'hospitalisation publique et privée (J.O.R.F., 25 avril 1996, p. 6311).

${ }^{104}$ Pour une étude générale du statut des ordonnances de l'article 38 voir : FAVOREU (L.), «Ordonnances ou règlements d'administration publique ?», R.F.D.A., 1987, pp. 686-699; voir également BOYER-MERENTIER (C.), Les ordonnances de l'article 38 de la Constitution du 4 octobre 1958, op. cit.

${ }^{105}$ Il est cependant précisé dans le Onzième rapport d'activité, précité, que le Premier Ministre a décidé d'assurer désormais la publication concomitante de ces tables.

${ }^{106}$ Commission supérieure de codification, Onzième rapport annuel (2000), précité.
} 
de temps obtenu par une entrée en vigueur différée permettra une information suffisante des usagers et contribuera à une meilleure compréhension du droit applicable.

Enfin la codification ne peut être poursuivie sans la participation active non seulement du Parlement qui a dépassé ses réticences en respectant les délais impartis par la loi d'habilitation, mais également des équipes au sein des ministères concernés qui sont désormais appelées à élaborer les parties réglementaires des codes ${ }^{107}$. En effet l'adoption des parties réglementaires donnera aux codes leur complète cohérence et améliorera la commodité des usagers et praticiens du droit. L'impulsion politique ayant présidé à cette relance depuis 1999 ne doit pas se démentir et au contraire doit se renforcer.

En outre, les codes ne resteront figés et évolueront en même temps que le droit applicable. Le maintien d'une veille juridique ayant pour objet d'incorporer aux codes les nouvelles dispositions des projets de loi s'avère nécessaire pour pallier les nombreuses difficultés à venir ${ }^{108}$. A cette fin le décret $n^{\circ} 2000-519$ du 16 juin $2000^{109}$ prévoit la consultation de la Commission supérieure de codification sur les projets de textes modifiant des codes existants. Conformément à ce décret, la Commission supérieure de codification a été saisie, pour la première fois, sur le projet de loi relatif à Mayotte ${ }^{110}$. Ce dernier apportait des modifications à plusieurs codes. La Commission s'est ainsi prononcée sur des questions relatives à la technique de codification et notamment sur le maintien de la cohérence rédactionnelle du Code général des collectivités territoriales.

Cet exemple prouve la collaboration existant entre les divers acteurs de la codification et la volonté des pouvoirs publics de la maintenir.

Désormais rien ne semble pouvoir entamer le déroulement harmonieux de l'entreprise de codification, fort précieuse à l'Etat de droit. Les critiques, nécessaires et les difficultés, inévitables, ne manqueront pas mais l'existence même d'un processus de codification restauré invite à l'optimisme ${ }^{111}$.

\footnotetext{
${ }^{107}$ On soulignera que la publication des parties réglementaires de trois codes ont été réalisées au cours de l'année 2000 : le Code général des collectivités territoriales, le Code des juridictions financières et le Code de justice administrative.

108 Certains auteurs soulignent les incertitudes dans la connaissance du droit applicable avec notamment l'exemple des « dispositions-balais » : MOYSAN (H.), «L'accessibilité et l'intelligibilité de la loi. Des objectifs à l'épreuve de la pratique normative », A.J.D.A., 2001, pp. 428-437.

109 Décret $\mathrm{n}^{\circ} 2000-519$ du 16 juin 2000 modifiant le décret $\mathrm{n}^{\circ} 89-647$ du 12 septembre 1989 relatif à la composition et au fonctionnement de la Commission supérieure de codification, J.O.R.F., 17 juin 2000 , p. 9111.

${ }^{110}$ La procédure législative aboutira à la loi ${ }^{\circ}$ 2001-616 du 11 juillet 2001 relative à Mayotte, J.O.R.F., 13 juillet 2001, p. 11199.

${ }^{111}$ Outre l'élaboration des parties réglementaires des codes publiés en l'an 2000, la Commission supérieure de codification souhaite entreprendre la rédaction des parties législatives des codes suivants : le Code de la recherche, le Code de l'administration, le Code du patrimoine, le Code de l'organisation judiciaire, le Code de l'entrée et du séjour des étrangers, le Code de la défense, le Code général de la fonction publique, le Code du tourisme et le Code général des transports.
} 